\title{
Particle Filter Based Robot Self-localization Using RGBD Cues and Wheel Odometry Measurements

\author{
Enyang Gao ${ }^{1, a^{*}}$, Zhaohua Chen ${ }^{1}$ and Qizhuhui Gao ${ }^{1}$
}

${ }^{1}$ School of information and control engineering, Shenyang Jianzhu University, Shenyang, China

aarnold0110@sina.com

Keywords: Mobile robot; Self-localization; RGBD; Wheel odometry; Particle filter

\begin{abstract}
Mobile robot localization in the GPS denied environments is increasingly exerting fundamental roles in a wide range of applications such as SFM and SLAM. However, the traditional single sensor based positioning methods are either unreliable or inaccurate in the long term. This paper presents a novel moving agent localizing approach that combines both RGBD cues and wheel odometry measurements within the particle filter based probabilistic framework. Unlike the traditional RGBD localization methods which are computationally expensive and non-robust, we took advantage of wheel odomery measurements as the prior information or say the initial values during the RBGD pose optimization process. Additionally, the optimal pose derived from visual sensor is, in turn, able to determine the reliability of the wheel odometry inputs. This verifying process is considerably useful in the presence of wheel slip. Experimental results validate that our approach is effective and reliable in wheel robot localization.
\end{abstract}

\section{Introduction}

Mobile robot localization determines the process of the location in unknown environments and it is the core toward the realization of automatic mobile robot navigation ability. In recent years, the use of particle filter algorithm [1,2] has become a hot topic in the robot autonomous positioning.

With respect to various types of sensors, positioning methods can be divided into discrepant categories such as the visual based, laser based, wheel based, etc. At present, sensors, such as odometer sensor, ultrasonic sensor, laser sensor and visual sensor, are widely used. Ultrasonic sensor and laser sensor due to their single sensing mode and long induction period have basically been taken as auxiliary positioning sensors; odometer is primarily designed on the basis of the wheeled mobile robot localization; it estimates the moving agent distance process by means of incrementally integrating the wheel encoder data in some special circumstances (smooth ground, topography inequality). However, the encoder values obtained are by and large inaccurate, and the positioning errors could accumulate in the long run. In this paper, the depth and visual cues are adopted. Complementary to RGB sensor which can uniquely provide the world point color information, depth sensor is able to sense the additional depth information. Combining color and depth cues, the feature point extraction time is significantly reduced. But compared with the extraction information speed, odometer is still slightly fasted. For better use of the information awareness by the sensor, the target position of the mobile robot accurately estimate under the indoor environment, using particle filtering fusion odometer and depth perception of environmental information by visual sensor can realize the independent position of mobile robot, and is verified by experiments[3, 4,5$]$.

\section{Sensor Model}

Odometer Model and Positioning Principle. This paper chooses two wheels differential driving wheeled mobile robot. Odometer sensor periodic read pulse number of photoelectric encoder, which is installed on the motor shaft. According to each read pulse, it can identify the current position of robot through distance and angle of the pulse $[6,7]$.

Odometer can detect driving wheel changed angle within some time through the photoelectric encoder be installed on the motor shaft. Assume that the driving wheel radius are $r$, the resolution 
of encoder is $w$ line, the reduction ratio of deceleration motor is $i_{s c}$, encoder output pulse $n$ in $\Delta t$ time for the wheel moving distance of $d s$ :

$$
d s=\frac{2 \pi n r}{w i_{s c}}
$$

Assume that the mobile robot driving wheel of left and right moving distance $d s_{L}$ and $d s_{R}$ in $\Delta t$ time respectively. And the driving wheel spacing distance is $l$, thus robot walk the distance and angle in $\Delta t$ time as shown below:

$$
\left\{\begin{array}{l}
s=\frac{d s_{L}+d s_{R}}{2} \\
\Delta \alpha=\frac{d s_{R}-d w_{L}}{l}
\end{array}\right.
$$

The mobile robot's trajectory calculation formula:

$$
\left\{\begin{array}{l}
x_{n}=x_{0}+\sum_{i=0}^{n-1} s_{i} \cos \alpha_{i} \\
y_{n}=y_{0}+\sum_{i=0}^{n-1} s_{i} \sin \alpha_{i} \\
\alpha_{n}=\alpha_{0}+\sum_{i=0}^{n-1} \Delta \alpha_{i}
\end{array}\right.
$$

The formula (3), $s_{i}$ show the robot moving distance from $i$ to $(i+1)$ time, $\Delta \alpha_{i}$ show the robot change in direction from $i$ to $(i+1)$ time, $\left(x_{i}, y_{i}, \alpha_{i}\right)$ show the robot location in $i$ time sampling. Moving trajectory is shown in Fig. 1.

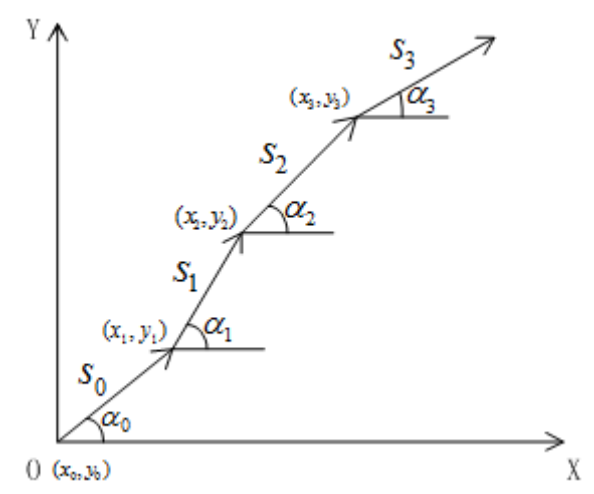

Figure 1. Moving trajectory

Depth Vision Sensor Model. This paper use the depth vision sensor based on kinect model. Depth vision sensor can sense abundant environmental information in order to estimate precision of mobile robot positioning [8]. Through depth vision sensor to obtain cues that with depth information. Namely the color image and it's corresponding the depth image.

\section{Feature Extraction and Processing Based on RGBD}

Fast Matching Algorithm Based on A Revised Four-Point Coplanar. The core idea of four-point coplanar fast matching algorithm: the known 3D point set $\mathrm{P}$ and offline processing of the 3D point set, then to calculate the normal vector of each point in point set $\mathrm{P}$; the offline data contains the 3D 
coordinates $(x, y, z)$ of the each point in the point set $\mathrm{P}$ 、 the depth information $d$ of each point, and the normal vector $\vec{n}$ of each point. Then real-time access to a frame image corresponding to the $3 \mathrm{D}$ point set $\mathrm{Q}$, the point set $\mathrm{Q}$ will be carried out in accordance with the point set $\mathrm{P}$. The matching stage: firstly, it can be selected that the four-point approximate coplanar base set B randomly, then it can be find that all the four point set $\mathrm{U}_{i}$ of approximate congruent with the base set $\mathrm{B}$ in point set $\mathrm{Q}$, next the rigid transformation $\mathrm{T}_{i}$ can be calculated between the set $\mathrm{B}$ and the set $\mathrm{U}_{i}$. Then the rigid transformation $\mathrm{T}_{i}$ apply to the whole set $\mathrm{P}$ and look for the best rigid transformation $\mathrm{T}^{*}$ that meet the threshold condition. Finally, the rigid transformation $\mathrm{T}^{*}$ can describe the space position relation [9] between two 3D space point set $\mathrm{P}$ and $\mathrm{Q}$. Four-point coplanar fast matching algorithm flow chart is shown in Fig. 2.

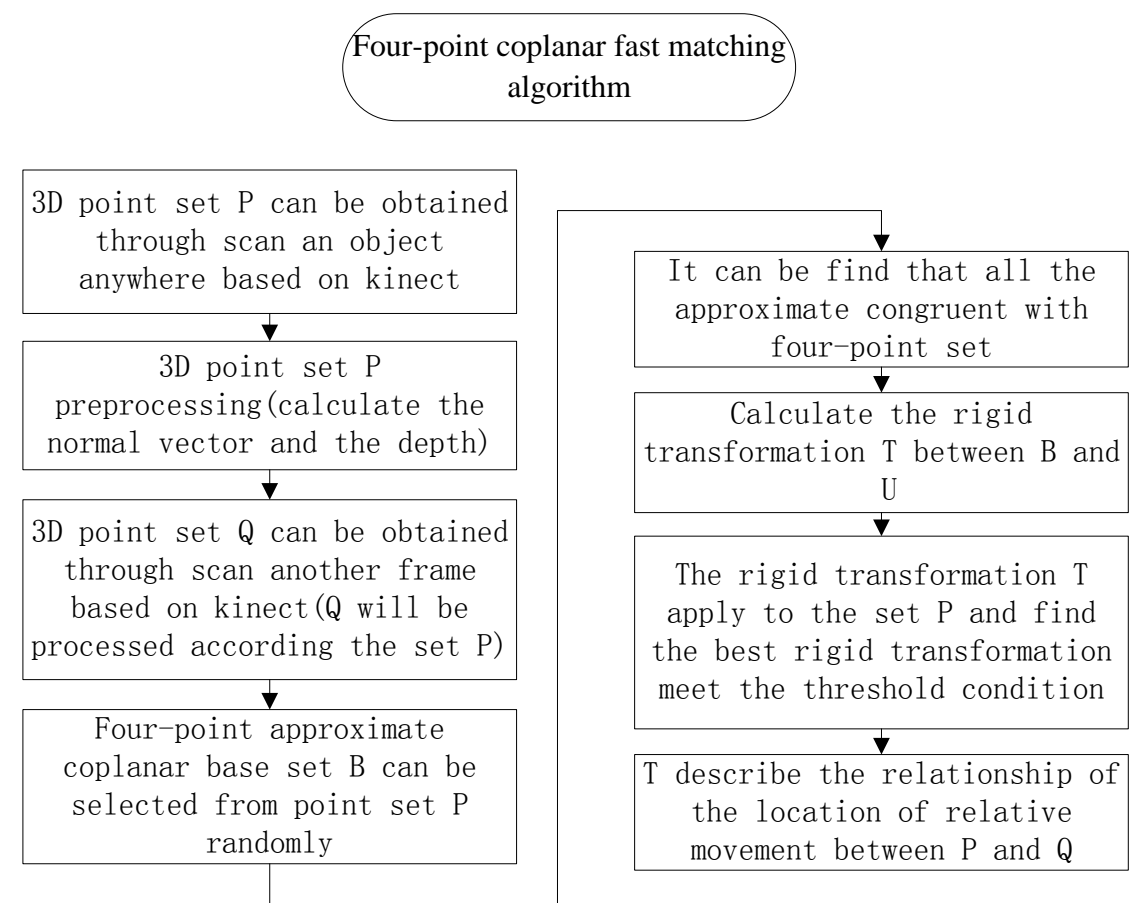

Figure 2. Four point coplanar fast matching algorithm flow chart

RGBD Cues Processing. Visual odometer technology based on kinect sensor is the core to achieve positioning technology. Flow chart shown in Fig. 3, it mainly includes three links:

Image Feature Extraction and Matching. Visual odometer technology based on kinect sensor is the image with depth information is processed, in other word, the three-dimensional image corresponding 3D point set can be feature extraction and matching. This article uses the four-point coplanar fast matching algorithm to make image extraction and matching. According the rules to extract the feature point set from a frame image, the next step is to match the next frame image according extract the feature points. Accuracy degree of feature matching will directly affect accuracy of the camera itself motion estimation. Using the least square method as feature point's standard of similarity. Specific operation method is calculate the rigid transformation through feature point set, and the 3D point set apply the rigid transformation. Then using the least square method to measure similarity between two 3D point set, thus get the best rigid transformation, so that it can be accurately estimate that the relationship of position of the camera movement.

Preliminary Motion Estimation. The 3D coordinates between two frames, through extraction and matching of feature point project to the ground and point sets in map and then to get the motion parameters in the camera itself. Camera motion parameters can express as the rigid transformation $(r, t), t$ represent translation matrix, $r$ represents the rotation matrix. Then the relationship between the feature point's coordinates and the movement parameters can be described by formula: 


$$
\mathrm{Q}=\mathrm{rP}+\mathrm{t}
$$

In formula (4) $P$ and $Q$ represent the $2 \mathrm{D}$ coordinates of feature point set that the current frame and the next frame image respectively. And $r$ represents the rotation matrix, $t$ represent translation matrix. Using Four-point coplanar fast matching algorithm to image matching for motion parameters, in other word, that is the rotation and translation matrix between two frame position relations, and it can be motion estimation by rotation and translation matrix on the camera itself.

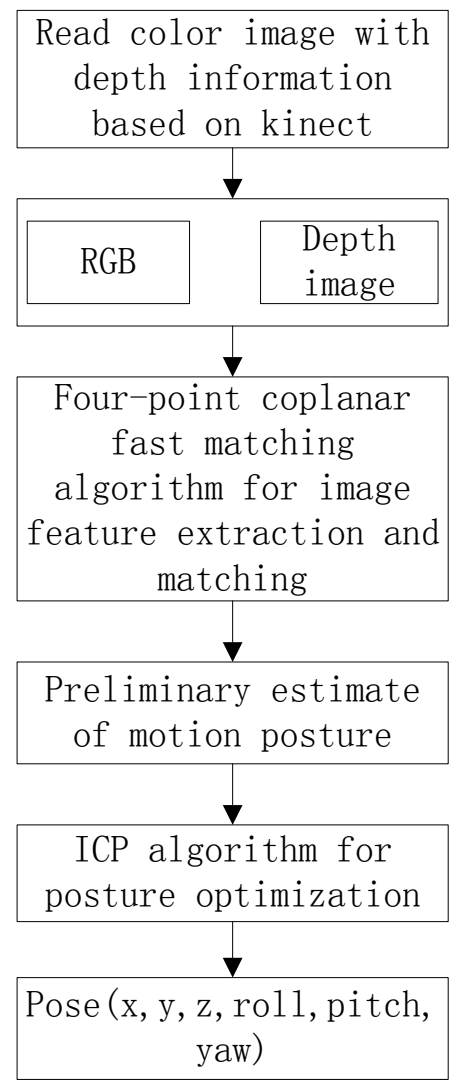

Figure 3. Flow chart of visual odometer based on Kinect

Movement Posture Optimization. Four-point coplanar fast matching algorithm is adopted to estimation initially can get a coarse matching values, rather than the optimal position estimation, so the pose estimation is not accurate just on the basis of rough matching[10]. Set a threshold in visual odometer based on Kinect sensor. According to the actual matching accuracy to determine whether or not to use the ICP algorithm further precise matching, thus realize movement pose estimation optimization.

\section{Particle Filter Localization Algorithm}

Particle filter positioning ideas is a probabilistic localization method based on Monte Carlo method, through the sensory information from the sensors recursive estimate pose probability density distribution of the state space and then to realize positioning. The key thought of Particle filter algorithm is to us a random sample of $n$ with weights express the credibility Bel(l) of the pose of the robot itself, and the sample space can express as $\mathrm{S}=\left\{s_{i} \mid i=1,2, \ldots, \mathrm{n}\right\}$. The discrete state sample collection can represent the reliability of the robot actual location. Each sampling $s_{i}$ consists of the robot's position $l_{i}=\left(x_{i}, y_{i}, \alpha_{i}\right)$ and the weights $p_{i}$, denoted by $s_{i}=\left(l_{i}, p_{i}\right)$. Which the probability of $p_{i}$ said robot is located in the location, and meet the conditions of $\sum_{i=1}^{N} p_{i}=1$. Particle filter positioning is two processes that based on both the motion model 
updating and the model perception updates.

Forecast Update Based on the Odometer. This paper chooses two wheels differential driving wheeled mobile robot, set $t$ moment robot posture $l_{t}=\left(x_{t}, y_{t}, \alpha_{t}\right)$ in the coordinate system, $l_{t+1}$ at the time $\mathrm{t}+1$, thus to establish the motion model of the robot is as follow:

$$
\left[\begin{array}{l}
x_{t+1} \\
y_{t+1} \\
\alpha_{t+1}
\end{array}\right]=\left[\begin{array}{l}
x_{t} \\
y_{t} \\
\alpha_{t}
\end{array}\right]+\left[\begin{array}{ll}
\cos \alpha_{t} & 0 \\
\sin \alpha_{t} & 0 \\
0 & 1
\end{array}\right] u_{t}+v_{t}
$$

The formula (5), $u_{t}=\left(s_{t}, \alpha_{t}\right)^{T}$ is the model input of odometer, $s_{t}, \alpha_{t}$ is the displacement of the mobile robot and the turning angle in $(\mathrm{t}, \mathrm{t}+1)$ time. And $v_{t}$ is the input noise subject to Gaussian white noise in the process of distribution.

Robot completed the prediction process of particle collection by the motion model of the robot:

$$
q_{t+1}=p\left(l_{t+1} \mid l_{t}, u_{t}\right) \operatorname{Bel}\left(l_{t}\right)
$$

The formula (6), $p\left(l_{t+1} \mid l_{t}, u_{t}\right)$ is the motion model, $q_{t+1}$ is the updated sampling distribution.

The Updated Perception Based on the Depth Visual. The particle set can make weights again by perception model in particle filter method. The perception data is through extract features of frame position to gain feature vector in the locating method based on depth visual. Matching success between two frames corresponding feature point set that the same object located in different position is the premise of solving image motion transformation model parameters. Assume that the robot obtain the actual observation environment model is the image $I_{k}$ in the current position. Then the updated sampling $s_{i}$ weight is:

$$
p_{v t}^{i}\left(z_{v t} \mid l_{v t}\right)=\frac{1}{N} \sum_{j=1}^{N} d\left(I_{k}, I_{j}\right)\left(D-\operatorname{dist}\left(I_{i}, I_{j}\right)\right)\left(\Phi-\delta\left(\theta_{i}, \theta_{j}\right)\right)
$$

The formula (7), the matching factor is $p_{v t}^{i}\left(z_{v t} \mid l_{v t}\right)$, and it represent the matching degree between the current observation model and after the updated sampling. $z_{v t}$ show the actual observation of the camera. $d\left(I_{k}, I_{j}\right)$ show the line distance between sampling images and the position of sampling $s_{i}$. $\Phi$ show preset maximum relative angle. $\delta\left(\theta_{i}, \theta_{j}\right)$ show the relative angle between the advanced sampling and sampling $s_{i}$.

Perception Update of Fusion Heterogeneous Sensor Information. Mobile robot can make the prediction and update of particle through the motion model, then it can update particle through probability model of depth visual sensor[6]. The important factor of measure heterogeneous sensor information is $p\left(z_{t} \mid l_{t}\right)$, and it represents the matching degree between the current observation and movement updated sampling, thus it can more accurate and reliable estimate the position of mobile robot in t time.

\section{The Experimental Results and Analysis}

Set up the Experimental Platform. An experiment was conducted to assess the accuracy of robot localization estimation within a typical environment in a lab room. The author set up the experimental platform as shown in Fig. 4, the computer use Inter Core i7 four core and eight thread processor, 4GB memory, run on Linux based on 64 bit ubuntu 14.04 system. 


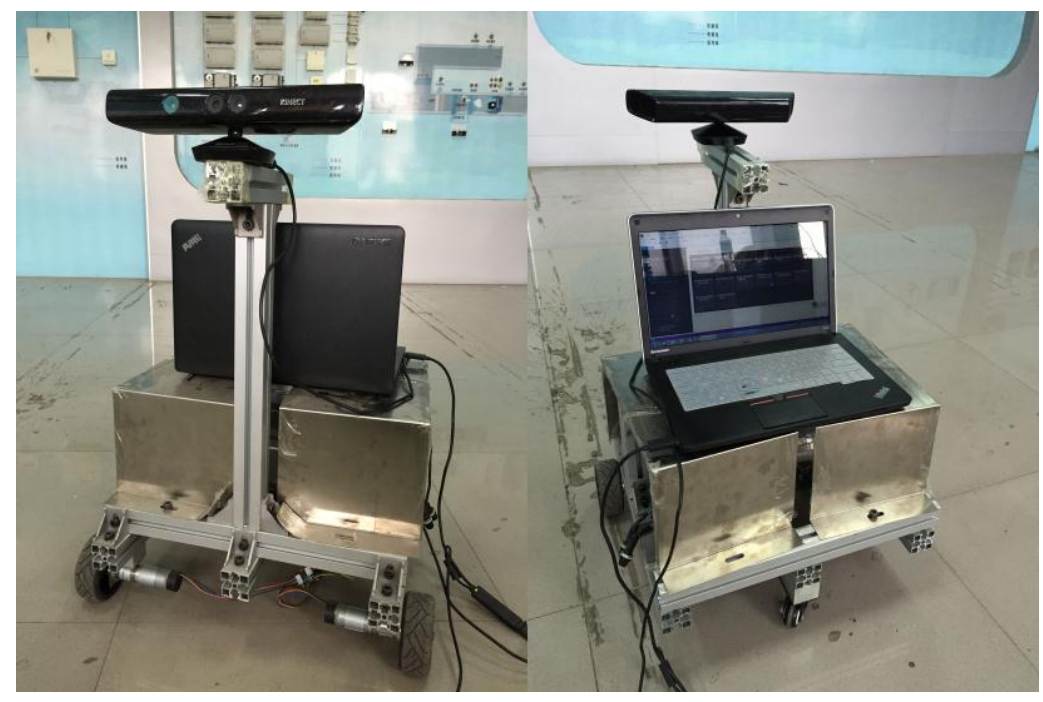

Figure 4. The experimental platform

Accuracy of the Trajectory Estimation. In this section, we will present our results on the accuracy of our robot system. The method is fairly accurate and they are show in Table 1.

We take 30 points in the trajectory and evaluate the accuracy of the trajectory estimation. The accuracy of the robot trajectory estimation is affected by velocity. With the increase of the robot's movement speed, the accuracy of trajectory estimation is reduced. This can be explained as, with the increase of the kinetic energy, the error of positioning estimation by wheel has decreased.

Table 1 Accuracy evaluation of trajectory with respect to velocity

\begin{tabular}{ccc}
\hline \hline speed & Translation RMSE & Rotation RMSE \\
\hline $0.05 \mathrm{~m} / \mathrm{s}$ & $0.153 \mathrm{~m}$ & $4.23^{\circ}$ \\
$0.15 \mathrm{~m} / \mathrm{s}$ & $0.191 \mathrm{~m}$ & $4.54^{\circ}$ \\
$0.30 \mathrm{~m} / \mathrm{s}$ & $0.211 \mathrm{~m}$ & $5.87^{\circ}$ \\
\hline
\end{tabular}

\section{Conclusion}

This paper proposes a particle filter based robot self-localization method using RGBD cues and wheel odometry measurements. In an environment which has been previously explored, this approach matches the point cloud project to the ground to the map. As a next step, we plan to add laser sensor to get more accurate robot motion trajectories.

\section{References}

[1] Luo F, Du B, Fan Z. Mobile robot localization based on particle filter[C]//Intelligent Control and Automation (WCICA), 2014 11th World Congress on. IEEE, 2014: 3089-3093.

[2] Wang Z, Tan J, Sun Z. Error Factor and Mathematical Model of Positioning with Odometer Wheel [J]. Advances in Mechanical Engineering, 2015, 7(1): 305981.

[3] Tsai G J, Chiang K W, Chu C H, et al. the Performance Analysis of AN Indoor Mobile Mapping System with Rgb-D Sensor [J]. The International Archives of Photogrammetry, Remote Sensing and Spatial Information Sciences, 2015, 40(1): 183.

[4] Aiger D, Mitra N J, Cohen-Or D. 4-points congruent sets for robust pairwise surface registration[C]//ACM Transactions on Graphics (TOG). ACM, 2008, 27(3): 85.

[5] Chi W, Zhang W, Gu J F, et al. A vision-based mobile robot localization method [C]//Robotics and Biomimetics (ROBIO), 2013 IEEE International Conference on. IEEE, 2013: 2703-2708. 
[6] Chen Z L. Depth Camera-Assisted Indoor Localization Enhancement [J]. 2013.

[7] Li J, Cheng $\mathrm{C} \mathrm{K}$, and Jiang $\mathrm{T} \mathrm{Y}$. Wavelet de-noising of partial discharge signals based on genetic adaptive threshold estimation [J]. IEEE Transactions on Dielectrics and Electrical Insulation, 2012, 19(2): 543-549.

[8] Stefan W, Chen K W, Guo H B, et al. Wavelet-based de-noising of positron emission tomography scans [J]. Journal of Scientific Computing, 2012, 50(3): 665-677.

[9] Zhu R., Zhou Z. A real-time articulated human motion tracking using tri-axis inertial/magnetic sensors package [J]. IEEE Transactions on Neural Systems and Rehabilitation Engineering, 2004, 12(2): 295-302.

[10]Mitianoudis N, Stathaki T. Pixel-based and region-based image fusion schemes using ICA bases [J]. Information Fusion, 2007, 8(2), 131-142. 